Neurosurg Focus 19 (2):E4, 2005

\title{
Technical strategies to approach aneurysms of the vertebral and posterior inferior cerebellar arteries
}

\author{
Niklaus Krayenbühl, M.D., Carlos A. Guerrero, M.D., and Ali F. Krisht, M.D. \\ Department of Neurosurgery, University of Arkansas for Medical Sciences, Little Rock, Arkansas
}

\begin{abstract}
Object. Aneurysms of the vertebral artery (VA) and proximal posterior inferior cerebellar artery (PICA) are rare and challenging lesions, as they are located in front of the brainstem and surrounded by the lower cranial nerves. Many different approaches have been described for their treatment, and have yielded different results. With the use of different examples of lesions, the authors describe their surgical strategy in the management of VA and PICA aneurysms.

Methods. The far-lateral approach was used, and the potential of its different extensions according to the specific anatomical location and configuration of different types of aneurysms is emphasized.

Conclusions. With the present knowledge of the microsurgical anatomy in the region of the foramen magnum, the far-lateral approach can be tailored to the specific anatomical and morphological configuration of an aneurysm in this region with good surgical results.
\end{abstract}

\section{KEY WORDS • aneurysm • far-lateral approach • skull base approach • foramen magnum - posterior inferior cerebellar artery • vertebral artery}

Aneurysms involving the region of the foramen magnum and the lower clivus include saccular, fusiform, and dissecting lesions of the VA; VBJ aneurysms; and proximal PICA aneurysms. Lesions in this area comprise up to $3 \%$ of intracranial aneurysms. ${ }^{23}$ Different approaches can be used to treat these lesions surgically, depending on their anatomical location and morphology. Traditionally, a paramedian suboccipital craniotomy was used..$^{10,27}$ Nevertheless, the closer the lesion is to the foramen magnum, or the farther away it is toward the midline, the more difficult it is to visualize adequately all the anatomical details of the region with the standard approaches without extending the craniotomy at the skull base level. Ventral approaches to this region were previously used for lesions close to the midline, but were later abandoned because of the significant morbidity associated with them. ${ }^{20,27}$ These earlier procedures were replaced with the far-lateral approach or one of its variants.

Different terminologies were used to describe the far-lateral approach to the VA and VBJ regions, depending on the extent of the bone removal. These terms include the lateral suboccipital, posterior suboccipital, posterolateral, dorsolat-

Abbreviations used in this paper: PICA = posterior inferior cerebellar artery; VA = vertebral artery; VBJ = vertebrobasilar junction. eral, far-lateral, extreme lateral, transcondylar, supracondylar, paracondylar, and transcondylar fossa approach..$^{2-4,6}$ $11,13,14,18,19,21,22,24,26,28$ To simplify the concepts related to approaches for aneurysms in this region, we describe the surgical-anatomical aspects involved in exposures for these lesions.

\section{CLINICAL MATERIAL AND METHODS}

We reviewed our experience with vascular lesions involving the VA and VBJ region in 10 patients. Based on this review, and guided by our experience with more than 50 others, neoplastic lesions involving the anterior lower third of the posterior fossa region, we describe a strategy that will help guide the extent of the approach needed, based on the anatomical details of the lesion.

\section{Intraoperative Neurophysiological Monitoring}

Intraoperative neurophysiological monitoring is routinely done in all patients and includes the use of somatosensory evoked potentials and brainstem auditory evoked potentials recorded bilaterally. The seventh, 10th, 11th, and 12th cranial nerves are monitored using electromyography, either unilaterally or bilaterally as needed. ${ }^{7}$ 


\section{Side of the Approach}

Aneurysms involving the VA are approached from the ipsilateral side. For aneurysms involving the VBJ, the approach and its extent are chosen based on several factors, including the location, size, and projection of the aneurysm; the presence or absence of subarachnoid hemorrhage; and the size and shape of the VA as well as the location and size of the sigmoid sinus and the jugular bulbs in some patients. For example, when dealing with a large, ruptured aneurysm it is best to plan the approach so that the dome is projecting away from the surgeon's view, to have direct access to the base of the lesion. On the other hand, when dealing with a small, unruptured aneurysm it may be easier to apply the clip with the fundus of the lesion in the surgeon's view. To achieve proximal control, it is preferable to approach the aneurysm from the side of a dominant VA. If the VAs are equal in size and symmetrical in location, the side of the nondominant jugular bulb may become a factor in deciding the side of the approach.

\section{Patient Positioning}

The positioning of the patient depends on several important factors, such as the planned approach, the lesion's properties, and the individual patient's characteristics. Elements of these factors include the location of the planned incision and craniotomy, the weight and height of the patient, and the length, thickness, and mobility of the patient's neck. In patients of average, normal weight who have a rather long neck and in whom a paramedian incision is planned, we use the prone position with a slight rotation of the neck toward the ipsilateral side. In patients who are obese and/or have a short, thick neck, we prefer the sitting position. This will help achieve better brain relaxation with the improved ventilation and venous drainage.

If the far-lateral approach is to be used, the patient is placed in the semilateral position, $45^{\circ}$ from horizontal. The head is kept in a neutral position to maintain the normal anatomical course of the VA. The shoulder is pulled and taped downward to avoid obstructing the access to the operating field. We use a slightly curved vertical incision 4 $\mathrm{cm}$ behind the mastoid bone and extending from the superior nuchal line down to the anterior border of the sternocleidomastoid muscle at the level of C-4, with anterior reflection of the skin flap. The most superficial layer of muscles is formed by the sternocleidomastoid and splenius capitis muscles. The muscles of the superficial layer are detached together from their occipital insertions and retracted inferiorly and anteriorly. In this manner the distance to the craniocervical junction is shortest compared with linear incisions, and the lateral access is not hindered by the retracted muscles, allowing surgery to be performed from a shorter distance with shorter instruments and with improved dexterity. On the other hand, this thick and wellvascularized flap can easily be reattached in closing, minimizing the risk of cerebrospinal fluid leaks.

If a bypass procedure is part of the plan, the occipital artery is preserved by dissecting the muscles layer by layer. The transverse process of the atlas is palpated and exposed approximately $1 \mathrm{~cm}$ below the tip of the mastoid and through the suboccipital triangle, which is formed medially by the rectus capitis posterior major muscle, superolaterally by the superior oblique, and inferolaterally by the inferior oblique muscle. It is in this triangle where the horizontal segment of the VA usually runs, within a groove on the lamina of the atlas. After exposing the suboccipital triangle, the complex venous compartment around the VA complex (the suboccipital cavernous sinus) can be visualized. ${ }^{1,5,25}$ Dissection of the region of the suboccipital cavernous sinus can cause an intimidating, brisk venous bleeding, which nowadays can easily be managed by injecting 1 $\mathrm{ml}$ of Tisseal through a small window, leading to a dramatic decrease in the venous oozing. This allows the dissection of the VA to proceed without the previously encountered difficulty. The VA complex can then be skeletonized extradurally, under full visualization, and mobilized if needed. The C-1 and C-2 roots are then identified. The ventral ramus of the $\mathrm{C}-2$ root crosses over the vertical segment of the VA between the atlas and the axis and should be preserved. The root of $\mathrm{C}-1$ can be sectioned for better exposure, especially when the VA is mobilized. The dural entry of the VA is then identified by using its muscular branch as a landmark, as the branch runs medially, just before the VA pierces the dura mater.

\section{Craniotomy Procedures}

The three variations of the craniotomy are as follows, and are chosen according to different segments of the VA (Figs. 1 and 2). 1) For a distally located PICA aneurysm, and/or lesions involving the middle third segment of the VA, the craniotomy is the one used with the standard paramedian suboccipital approach. The craniotomy extends from the midline, incorporating the posterolateral aspect of the foramen magnum and with its inferior extension flush with the skull base region (Figs. 2 and 3). 2) For a PICA aneurysm arising from the proximal one third segment of the VA (Figs. 2 and 3, Video 1), the aforementioned craniotomy is further extended inferiorly. On the lateral aspect of this extension, the superomedial part of the condyle is drilled and removed, until satisfactory visualization of the VA proximal

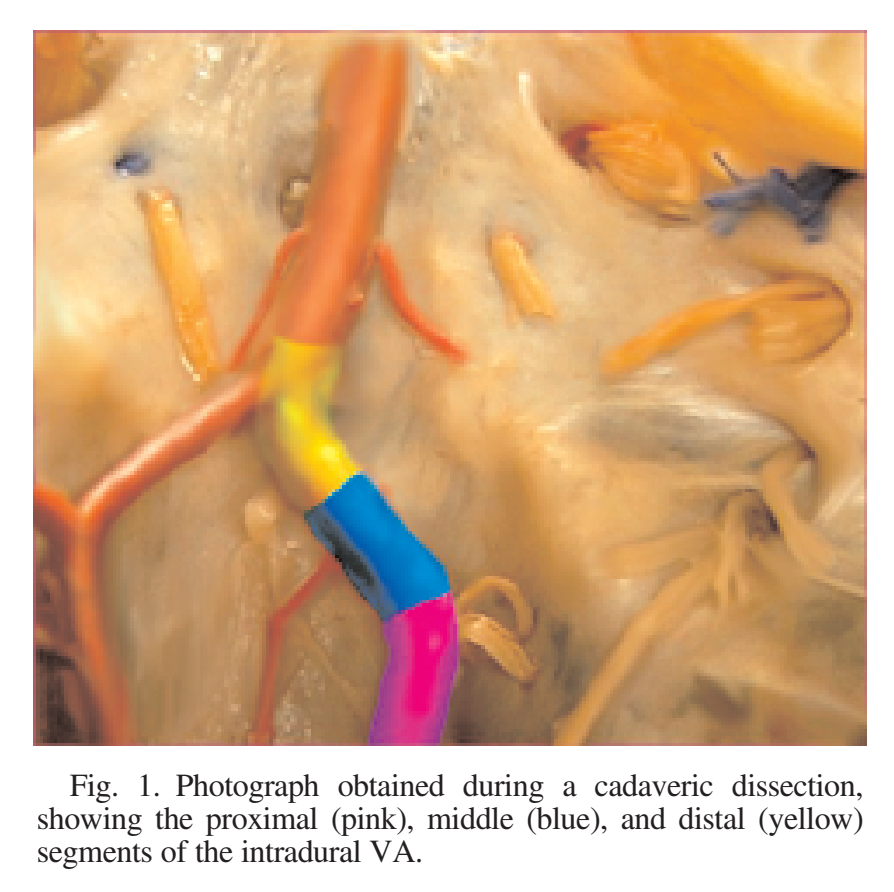




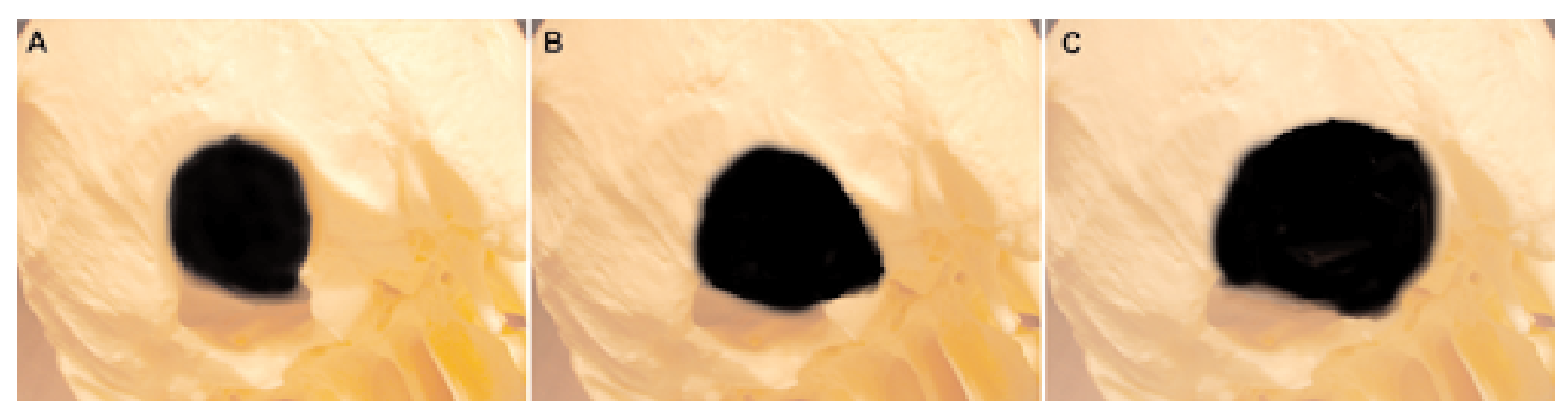

Fig. 2. Inferior views of the posterior skull base showing the extent of bone removal as it relates to the location of the aneurysm origin from the VA. A: Lateral suboccipital craniotomy (recommended for aneurysms of the middle segment of the VA; see Fig. 3A). B: Extended lateral suboccipital craniotomy with additional drilling of the superomedial aspect of the condyle (recommended for aneurysms of the proximal segment of the VA; see Fig. 3B and Video 1). C: Far-lateral craniotomy with further removal of the condyle and drilling of the mastoid to skeletonize the sigmoidal sinus down to the jugular bulb region (recommended for aneurysms of the distal segment of the VA and the VBJ; see Fig 3C and Video 2).

to the aneurysm base is achieved. The view may be further enhanced by removing the ipsilateral half of the arch of C1 , which enables a view through the operating microscope from a medial-to-lateral direction. 3) When the far-lateral approach is needed, as when dealing with aneurysms of the VBJ region, the suboccipital craniotomy is extended from the midline medially to the sigmoidal sinus laterally, incorporating the foramen magnum (Figs. 2 and 3, Video 2). The mastoid is opened to skeletonize the sigmoidal sinus fully down to its junction with the jugular bulb, so that these structures can be partially mobilized to gain more exposure laterally.

Video 1. Microscopic views obtained in a 70-year-old woman with history of syncope. As part of her workup she was found to have a VA aneurysm arising at the origin of the PICA, which originated from the proximal segment of the VA. This location necessitated partial drilling of the condyle to achieve better proximal control for safer clip placement to occlude the aneurysm. (Click here to view with Windows Media Player, or here to view with RealPlayer.)

Video 2. Microscopic views obtained in a 52-year-old woman with a previously clip-occluded, ruptured posterior communicating artery aneurysm, and in whom an incidental finding of a VBJ aneurysm was made. The patient selected clip occlusion as her treatment option for the second lesion. The exposure was a far-lateral one, enhanced by skeletonizing the sigmoidal sinus to the level of the jugular bulb. (Click here to view with Windows Media Player, or here to view with RealPlayer.)

When exposing and drilling part of the condyle, an important landmark for orientation is the exit site of the condylar vein from the posterior condylar canal. ${ }^{18}$ This vein, which can be a source of profound bleeding, should be coagulated and cut. The occipital condyle is situated below

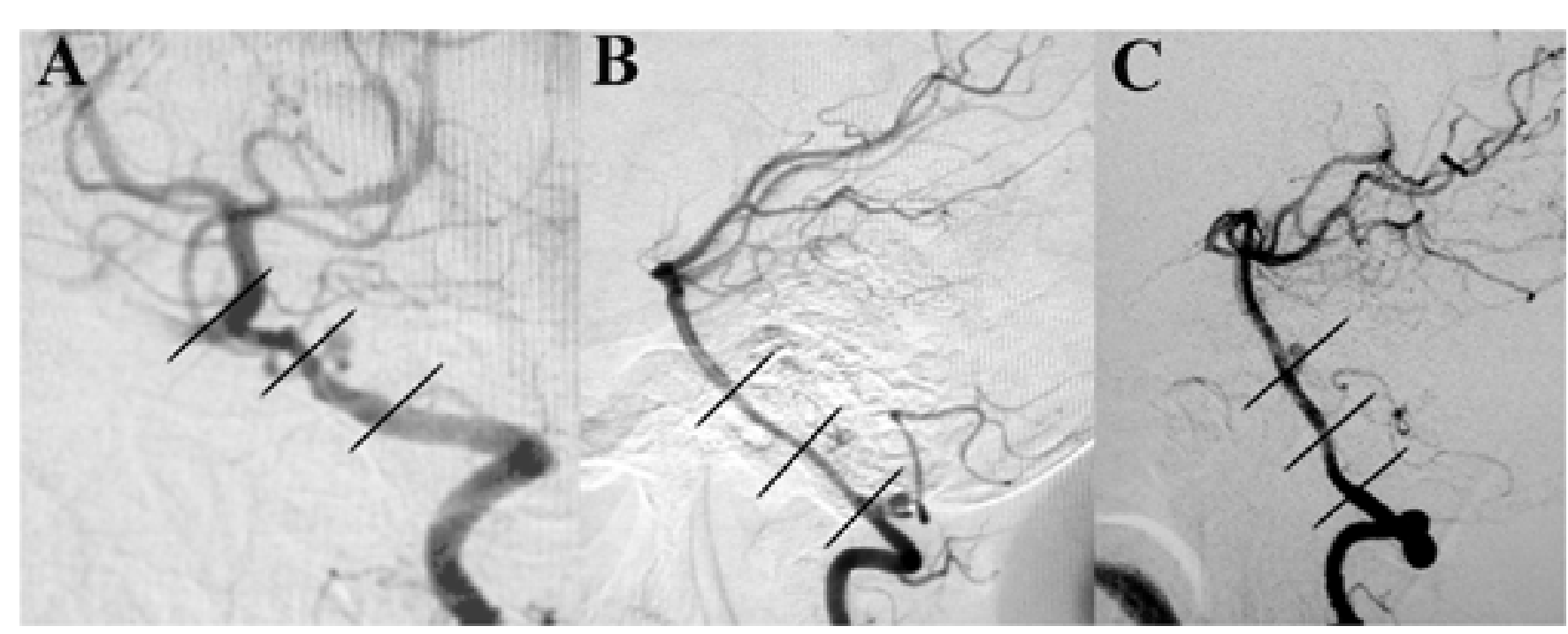

Fig. 3. Angiograms of VA aneurysms originating from the middle (panel A, PICA aneurysm), proximal (panel B, PICA aneurysm), and VBJ (panel C) segments of the VA. Black lines designate the proximal, middle, and distal segments of the artery. 
and the jugular tubercle is above the condylar canal. The jugular tubercle may sometimes be removed to enhance the view anterior to the lower cranial nerves.

\section{Intradural Step}

After opening the dura mater, it is important to take ample time to identify the different neurovascular structures and to understand both the normal and the diseased anatomy. A good knowledge of the anatomical variations is an important contributor to the safety of any operation. ${ }^{9,12,}$ ${ }^{15-17}$ For example, care should be taken to identify the posterior spinal artery, which can originate from the VA extradurally. ${ }^{8,26}$

After opening the dura mater, cerebrospinal fluid is drained from the cisterna magna to relax the brain. In the region of the proximal segment of the VA, cutting the dentate ligament can help mobilize the artery, allowing the base of the aneurysm to be better visualized from more than one direction. Extra care is needed when handling the lower cranial nerves, to prevent postoperative swallowing difficulties. In the majority of lesions, the nerves are preserved and there is no need to cut any of the branches. No matter how small and flimsy those branches look, as important as their functions are, they should always be handled with care and preserved. Many times they may be mistaken for arachnoid or fibrous bands. To avoid such mistakes, it is important to familiarize oneself with the normal anatomy by studying cadavers.

After dissecting the base of the aneurysm, a decision is made regarding the clip application process. It is important to visualize both the proximal and distal aspects of the VA, especially if the vessels on both sides are similar in size. We recommend applying a temporary clip before permanent clip placement for ruptured aneurysms. Occasionally one proximal clip is enough if the ipsilateral VA is dominant. This is supplemented with bipolar coagulation to obliterate the bleeding spot, and an attempt can be made to convert the case to an elective-like, nonruptured aneurysm operation. The surgeon can then relax and take as much time as necessary to apply the clip perfectly.

When dealing with VBJ aneurysms, the contralateral VA should be visualized for good proximal control to be achieved.

\section{CONCLUSIONS}

The extent of the lateral suboccipital approach should be tailored to the specific anatomical location and morphological configuration of vascular lesions involving the VA or PICA, while also taking into consideration the possible presence of subarachnoid hemorrhage. With an appropriate surgical strategy, visualization of the lesion can be improved, making the microsurgical treatment safer and thus leading to better surgical results.

\section{References}

1. Arnautovic KI, Al-Mefty O, Pait TG, et al: The suboccipital cavernous sinus. J Neurosurg 86:252-262, 1997

2. Babu RP, Sekhar LN, Wright DC: Extreme lateral transcondylar approach: Technical improvements and lessons learned. J Neurosurg 81:49-59, 1994

3. Bertalanffy H, Gilsbach JM, Mayfrank L, et al: Planning and surgical strategies for early management of vertebral artery and vertebrobasilar junction aneurysms. Acta Neurochir (Wein) 134:60-65, 1995

4. Bertalanffy H, Seeger W: The dorsolateral, suboccipital, transcondylar approach to the lower clivus and anterior portion of the craniocervical junction. Neurosurgery 29:815-821, 1991

5. Caruso RD, Rosenbaum AE, Chang JK, et al: Craniocervical junction venous anatomy on enhanced MR images: the suboccipital cavernous sinus. AJNR Am J Neuroradiol 20: 1127-1131, 1999

6. D'Ambrosio AL, Kreiter KT, Bush CA, et al: Far lateral suboccipital approach for the treatment of proximal posteroinferior cerebellar artery aneurysms: surgical results and long-term outcome. Neurosurgery 55:39-50, 2004

7. De Monte F, Warf P, Al-Mefty O: Intraoperative monitoring of the lower cranial nerves during surgery of the jugular foramen and lower clivus, in Loftus CM, Traynelis VC (eds): Intraoperative Monitoring Techniques in Neurosurgery. New York: McGraw-Hill, 1994, pp 205-212

8. de Oliveira E, Rhoton AL Jr, Peace D: Microsurgical anatomy of the region of the foramen magnum. Surg Neurol 24:293-352, 1985

9. Dowd GC, Zeiller S, Awasthi D: Far lateral transcondylar approach: dimensional anatomy. Neurosurgery 45:95-99, 1999

10. Drake CG, Peerless SJ, Hernesniemi JA: Surgery of Vertebrobasilar Aneurysms: London, Ontario Experience on 1767 Patients. Vienna: Springer-Verlag, 1995

11. Goel A, Desai K, Muzumdar D: Surgery on anterior foramen magnum meningiomas using a conventional posterior suboccipital approach: a report on an experience with 17 cases. Neurosurgery 49:102-106, 2001

12. Grand W, Budny J, Gibbons K, et al: Microvascular surgical anatomy of the vertebrobasilar junction. Neurosurgery 40: 1219-1223, 1997

13. Hernesniemi JA, Vapalahti MP, Niskanen M, et al: Management outcome in vertebrobasilar artery aneurysms by early surgery. Neurosurgery 31:857-861, 1992

14. Heros RC: Lateral suboccipital approach for vertebral and vertebrobasilar artery lesions. J Neurosurg 64:559-562, 1986

15. Hudgins RJ, Day AL, Quisling RG, et al: Aneurysms of the posterior inferior cerebellar artery: A clinical and anatomical analysis. J Neurosurg 58:381-387, 1983

16. Katsuta T, Rhoton AL Jr, Matsushima T: The jugular foramen: microsurgical anatomy and operative approaches. Neurosurgery 41:149-201, 1997

17. Lister JR, Rhoton AL Jr, Matsushima T, et al: Microsurgical anatomy of the posterior inferior cerebellar artery. Neurosurgery 10:170-199, 1982

18. Matsushima T, Matsukado K, Natori Y, et al: Surgery on a saccular vertebral artery-posterior inferior cerebellar artery aneurysm via the transcondylar fossa (supracondylar transjugular tubercle) approach or the transcondylar approach: surgical results and indications for using two different lateral skull base approaches. J Neurosurg 95:268-274, 2001

19. Nanda A, Vincent DA, Vannemreddy PS, et al: Far-lateral approach to intradural lesions of the foramen magnum without resection of the occipital condyle. J Neurosurg 96:302-309, 2002

20. Ogilvy CS, Barker FG II, Joseph MP, et al: Transfacial transclival approach for midline posterior circulation aneurysms. Neurosurgery 39:736-741, 1996

21. Perneczky A: The posterolateral approach to the foramen magnum, in Samii M (ed): Surgery In and Around the Brain Stem and the Third Ventricle. Berlin, Springer-Verlag, 1986, pp 460-466

22. Salas E, Sekhar LN, Ziyal IM, Caputy AJ, Wright DC: Variations of the extreme lateral craniocervical approach: anatomical study and clinical analysis of 69 patients. J Neurosurg 90 (Suppl 4):206-219, 1999 


\section{Technical strategies to approach aneurysms of the VA and PICA}

23. Salcman M, Rigamonti D, Numaguchi Y, et al: Aneurysms of the posterior inferior cerebellar artery-vertebral artery complex: Variations on a theme. Neurosurgery 27:12-21, 1990

24. Sen CN, Sekhar LN: An extreme lateral approach to intradural lesions of the cervical spine and foramen magnum. Neurosurgery 27:197-204, 1990

25. Takahashi S, Sakuma I, Omachi K, et al: Craniocervical junction venous anatomy around the suboccipital cavernous sinus: evaluation by MR imaging. Eur Radiol:Feb 27, 2005; [Epub ahead of print]

26. Wen HT, Rhoton AL Jr, Katsuta T, et al: Microsurgical anatomy of the transcondylar, supracondylar, and paracondylar extensions of the far-lateral approach. J Neurosurg 87:555-585, 1997

27. Yaşargil MG: Vertebrobasilar aneurysms, in Yaşargil MG (ed):
Microneurosurgery: Clinical Considerations, Surgery of the Intracranial Aneurysms and Results. Stuttgart: Georg Thieme, 1984, vol II, pp 270-295

28. Yonekawa Y, Kaku Y, Imhof HG, et al: Posterior circulation aneurysms: Technical strategies based on angiographic anatomical findings and the results of 60 recent consecutive cases. Acta Neurochir Suppl (Wien) 72:123-140, 1999

Manuscript received July 7, 2005.

Accepted in final form July 18, 2005.

Address reprint requests to: Niklaus Krayenbühl, M.D., Department of Neurosurgery, University of Arkansas for Medical Sciences, 4301 West Markham Street \# 501, Little Rock, Arkansas 72205. email: nkrayenbuehl@bluewin.ch. 